\title{
A checklist of 'Psocoptera' (Psocodea) from Brazil: an update to the list of 2009 of García Aldrete and Mockford, with an identification key to the families
}

\author{
Alberto Moreira da Silva-Neto ${ }^{1}$ \& Alfonso Neri García Aldrete ${ }^{2}$
}

\begin{abstract}
1 Instituto Nacional de Pesquisas da Amazônia (INPA), Coordenação de Pesquisas em Entomologia (CPEN), Programa de Pós-Graduação em Entomologia. Manaus, AM, Brasil. ORCID: http://orcid.org/0000-0002-4522-3756. E-mail: bio.alberto@gmail.com

${ }^{2}$ Universidad Nacional Autónoma de México (UNAM), Instituto de Biología, Departamento de Zoología, Laboratorio de Entomología. México, D.F., México. ORCID: http://orcid.org/0000-0001-7214-7966. E-mail: anga@ib.unam.mx
\end{abstract}

\begin{abstract}
The described species of Psocoptera currently known for Brazil are listed, with state distribution and biogeographic status. An identification key to the families recorded in Brazil is presented.
\end{abstract}

Key-Words. Geographic distribution; Psocids; Neotropics.

\section{INTRODUCTION}

Psocoptera have no popular name in Brazil, being known in other countries as book lice, bark lice or psocids. These insects are small, measuring from 1 to $10 \mathrm{~mm}$ in length and feed on algae, lichens, fungi and organic fragments (Smithers, 1991). Psocoptera is a paraphyletic group because the Phthiraptera are phylogenetically embedded in the Psocoptera infraorder Nanopsocetae (Johnson et al., 2004; Yoshizawa \& Johnson, 2010; Yoshizawa \& Lienhard, 2010). To maintain monophyly on the order level, the former orders Psocoptera and Phthiraptera now constitute the order Psocodea (Yoshizawa \& Johnson, 2006). As true lice and psocids have distinct habits, and are studied by different methods and by different experts, Psocoptera is often still treated as an order in the traditional way. Through the literature of 2014, Psocoptera includes 5,941 extant species, in 485 genera (Mockford, 2018). García Aldrete \& Mockford (2009) listed for Brazil 425 species (including undescribed ones) in 94 genera and 28 families.

The present paper provides an update to the 2009 list of García Aldrete and Mockford and an identification key to the families of Psocoptera recorded in Brazil.

\section{MATERIAL AND METHODS}

The updating of the information on the Brazilian fauna in García Aldrete \& Mockford
(2009) is derived mostly from the ongoing study of the vast Psocoptera collection in the Instituto Nacional de Pesquisas da Amazônia (INPA), in Manaus, Amazonas; from the study of the Psocoptera collected through the program PPBio-Semi-Árido, housed in the Entomological Collection Prof. Johann Becker of the Zoology Museum of the Universidade Estadual de Feira de Santana, in Feira de Santana, Bahia, Brazil (MZFS), from the study of the Psocoptera collected through the program Cave invertebrates in Brazil: threats, description of new taxa and definition of priority areas for conservation. Module I: Northeast Brazil, housed in the Invertebrate Collection of the Biology Department, Universidade Federal de Lavras, Minas Gerais, Brazil. Information was also extracted from the world catalogue of Lienhard \& Smithers (2002), the country checklists of Lienhard (2016), a catalogue of type specimens of Psocoptera (Insecta: Psocodea) deposited in the collection of the Museu de Zoologia da Universidade de São Paulo, São Paulo, Brazil (Silva-Neto et al., 2019a) and a list of Psocoptera species from Colombia (García Aldrete et al., 2018). In addition, from 2009 to date, numerous articles have been published with new species or new records of Brazilian species of Psocoptera and these papers were also used to update the list (Lienhard et al., 2010, 2012; Silva-Neto \& García Aldrete, 2012; García Aldrete \& Silva-Neto, 2013; Lienhard \& Lopes-Ferreira, 2013a, 2013b; SilvaNeto et al., 2013; García Aldrete \& Silva-Neto, 2014; Lienhard \& Lopes-Ferreira, 2014; Silva-Neto et al., 2014a, 2014b, 2015; Silva-Neto \& García Aldrete, 
2015; Román-P. et al., 2015, Lienhard \& Lopes-Ferreira, 2015; García Aldrete et al., 2016; Silva-Neto et al., 2016a, 2016b, 2016c; Alves-Oliveira et al., 2017; Yoshizawa et al., 2017; García Aldrete et al., 2018; Vinasco-Mondragón et al., 2018; Silva-Neto et al., 2018a, 2018b, 2018c, 2018d; García Aldrete \& Silva-Neto, 2019; Moura-Lima et al., 2019; Silva-Neto \& García Aldrete, 2019; Silva-Neto et al., 2019b; García Aldrete \& Silva-Neto, 2020).

In the species list after the name of each species and its author, the Brazilian states appear in parentheses. If the species is not endemic to Brazil, the name of the country in which it was registered is provided or if it has a very wide distribution it is treated as tropical waif, widespread in the American tropics, widespread in the tropics, Holarctic, widely distributed or Cosmopolitan. In the cases that after the name of each species appears only "Brazil" without delimiting the Brazilian state of occurrence, it was because this species was described without providing information on the collecting locality in Brazil.

\section{RESULTS}

The list presented below includes 460 species in 97 genera and 30 families, with distribution in Brazilian states.

\section{Trogiomorpha}

\section{Lepidopsocidae}

1. Nepticulomima brasiliensis (Enderlein). (Pará).

2. Notolepium brasiliense New. (Mato Grosso).

3. Proentomum personatum Badonnel. (Roraima). Tropical waif.

4. P. pulvillata (New). (Mato Grosso).

5. Soa flaviterminata Enderlein. (Amazonas, Pará, Roraima). Widely distributed.

6. S. violacea New. (Amazonas, Mato Grosso).

7. Echmepteryx angusta New. (Mato Grosso).

8. E. bishopi New. (Mato Grosso).

9. E. falco (Badonnel). (Amazonas, Bahia). Tropical waif.

10. E. fuscata New. (Mato Grosso).

11. E. lealae New. (Pernambuco).

12. E. lutosa Mockford. (Roraima).

13. E. madagascariensis (Kolbe). (Rio de Janeiro, Bahia). Tropical waif.

14. E. uniformis Mockford. (Roraima).

\section{Trogiidae}

15. Cerobasis guestfalica (Kolbe). (Pernambuco, Rio de Janeiro). Widely distributed.

\section{Psoquillidae}

16. Psoquilla marginepunctata Hagen. (Mato Grosso, Santa Catarina). Widely distributed.

17. Rhyopsocus rafaeli García Aldrete. (Amazonas).

\section{Psyllipsocidae}

18. Psocathropos lachlani Ribaga. (Alagoas, Amazonas, Bahia, Ceará, Espírito Santo, Minas Gerais, Sao Paulo). Widely distributed.

19. P. pilipennis (Enderlein). (Ceará, Piauí, Rio Grande do Norte). Widely distributed.

20. Psyllipsocus angustipennis Lienhard. (Minas Gerais).

21. P. clunioventralis Lienhard. (Mato Grosso).

22. P. clunjunctus Lienhard. (Bahia, Goiás, Minas Gerais, Rio Grande do Norte, Tocantins).

23. P. didymus Lienhard. (Mato Grosso).

24. P. falcifer Lienhard. (Minas Gerais).

25. P. fuscistigma Lienhard. (Ceará).

26. P. marconii Lienhard. (Minas Gerais).

27. P. proximus Lienhard. (Amazonas).

28. P. punctulatus Lienhard. (Piauí).

29. P. radiopictus Lienhard. (Alagoas).

30. P. ramburii Selys-Longchamps. (Espirito Santo, Minas Gerais, Rio Grande do Sul, São Paulo). Widely distributed.

31. P. serrifer Lienhard. (Bahia, Minas Gerais).

32. P. similis Lienhard. (Minas Gerais).

33. P. spinifer Lienhard. (Bahia, Ceará, Goiás, Mato Grosso, Minas Gerais, Piauí, Rio Grande do Norte, São Paulo).

34. P. subtilis Lienhard. (Rio Grande do Norte).

35. P. thaidis Lienhard. (Piauí).

36. P. yucatan Gurney. (Alagoas, Bahia, Ceará, Espírito Santo, Minas Gerais, Pará, Rio Grande do Norte, São Paulo). Widespread in the tropics.

\section{Prionoglarididae}

37. Neotrogla aurora Lienhard. (Tocantins).

38. N. brasiliensis Lienhard. (Minas Gerais).

39. N. curvata Lienhard \& Ferreira. (Bahia).

40. N. truncata Lienhard. (Bahia).

\section{Troctomorpha}

\section{Troctopsocidae}

41. Troctopsoculus brasiliensis New. (Mato Grosso). Mexico.

42. Troctopsocus separatus (Roesler). (Santa Catarina). Trinidad.

\section{Musapsocidae}

43. Musapsocus mockfordi New. (Amazonas).

44. M. newi Mockford. (Roraima).

\section{Amphientomidae}

45. Lithoseopsis brasiliensis García Aldrete, Silva Neto \& Lopes Ferreira. (Pará).

46. Seopsocus acuminatus Roesler. (Santa Catarina).

47. S. albiceps Mockford. (Roraima). 
48. S. fasciatus Mockford. (Roraima).

49. S. rafaeli Mockford. (Roraima).

50. S. rotundatus Roesler. (Santa Catarina).

\section{Liposcelididae}

51. Belaphopsocus badonneli New. (Mato Grosso). Colombia, Mexico, Paraguay.

52. Belaphotroctes brunneus Badonnel. (São Paulo).

53. B. ghesquierei Badonnel. (Mato Grosso, Roraima, Sao Paulo). Widespread in Tropical Africa and America.

54. B. major Badonnel. (Mato Grosso).

55. B. mimulus Badonnel. (São Paulo).

56. B. ocularis Badonnel. (São Paulo).

57. B. striatus Badonnel. (São Paulo).

58. B. vaginatus Badonnel. (São Paulo).

59. Chaetotroctes lenkoi Badonnel. (Mato Grosso).

60. Embidopsocopsis newi Badonnel. (Mato Grosso).

61. Embidopsocus brasiliensis Badonnel. (São Paulo).

62. E. flexuosus parvulus Badonnel. (Mato Grosso).

63. E. frater Badonnel. (São Paulo).

64. E. leucomelas (Enderlein). (Mato Grosso). Paraguay.

65. E. luteus Hagen. (Mato Grosso). Cuba, Mexico.

66. E. mendax Badonnel. (Mato Grosso, São Paulo). Argentina.

67. E. pilosus Badonnel. (Mato Grosso, São Paulo).

68. E. similis Badonnel. (Mato Grosso).

69. E. virgatus (Enderlein). (Mato Grosso, São Paulo). Argentina, Paraguay.

70. Liposcelis bostrychophila Badonnel. (Rio de Janeiro). Cosmopolitan.

71. L. entomophila (Enderlein). (Amazonas, Rio de Janeiro). Cosmopolitan.

72. L. fusciceps Badonnel. (São Paulo). Mexico.

73. L. lenkoi Badonnel. (São Paulo).

\section{Pachytroctidae}

74. Tapinella gamma Mockford. (Roraima).

75. T. maracana Mockford. (Roraima).

76. T. ornaticeps Mockford. (Roraima). Venezuela.

77. Antilopsocus nadleri Gurney. (D.F., Mato Grosso, Minas Gerais). Colombia, Trinidad.

78. Pachytroctes brasilianus Roesler. (Santa Catarina). Venezuela.

79. Psyllotroctes plaumanni Roesler. (Santa Catarina).

\section{Psocomorpha}

\section{Epipsocidae}

80. Epipsocus acanthus New. (Amazonas).

81. E. argutus New. (Amazonas).

82. E. atratus New. (Amazonas, Roraima).

83. E. badonneli Mockford. (Pará, Roraima). Colombia.

84. E. borgmeieri Karny. (Rio de Janeiro).

85. E. foliatus Mockford. (Roraima).

86. E. fuscareolatus New. (Amazonas).
87. E. latistigma Roesler. (Santa Catarina).

88. E. maculithorax New. (Amazonas).

89. E. manausensis García Aldrete \& Silva-Neto. (Amazonas).

90. E. meruleus New. (Amazonas).

91. E. pennyi New. (Amazonas).

92. E. pereirai Badonnel. (Amazonas, Mato Grosso, Rondônia).

93. E. phaeus New. (Amazonas, Roraima).

94. E. quurcus Roesler. (Santa Catarina).

95. E. roraimensis Mockford. (Roraima).

96. E. serenus Roesler. (Santa Catarina).

97. E. stigmaticus Mockford. (Roraima).

98. E. uniformis New. (Mato Grosso).

99. E. verrucosus New. (Amazonas).

100. E. willineri New. (Mato Grosso).

101. Goja nebulosa (Roesler). (Santa Catarina).

102. G. picta (Banks). "Brazil".

103. G. plaumanni (Roesler). (Paraná, Santa Catarina).

104. Mesepipsocus brasilianus (New). (Mato Grosso). Peru.

105. M. brasiliensis (New). (Amazonas). Colombia.

106. M. brevistigma (New). (Mato Grosso). Colombia. Peru.

107. M. brunellus (New). (Mato Grosso).

108. M. capitulatus (New). (Amazonas).

109. M. fuscatus (New). (Mato Grosso). Peru.

110. M. newi Badonnel. (São Paulo).

111. M. niger (New). (Mato Grosso). Peru.

112. M. obscurus (New). (Mato Grosso).

113. M. proctus (New \& Thornton). (Pará). Colombia. Peru.

114. M. roesleri (New). (Mato Grosso).

115. M. roncadorensis (New). (Mato Grosso).

116. M. semiclarus (Mockford). (Roraima).

117. M. sinuatus (New). (Mato Grosso). Peru.

118. M. taitubai (New). (Mato Grosso).

119. Neurostigma dispositum Roesler. (Amazonas, Mato Grosso, Santa Catarina, Sao Paulo). Mexico, Peru.

120. N. enderleini New. (Amazonas).

121. N. paucivenosum New. (Amazonas).

122. N. radiatum Mockford. (Roraima).

123. N. roesleri New. (Amazonas).

124. N. xanthopterum New. (Amazonas). Colombia.

125. Papillopsocus oriximinaensis García Aldrete. (Pará).

\section{Dolabellopsocidae}

126. Dolabellopsocus carcinus Mockford. (Roraima).

127. D. catenatus Mockford. (Roraima).

128. D. ctenatus (New). (Amazonas, Bahia, Mato Grosso). Peru.

129. D. eertmoedi Badonnel. (Mato Grosso).

130. D. flavipennis (Roesler). (Santa Catarina).

131. D. intermedius Eertmoed. (Pará, Rondônia). Trinidad.

132. D. lobatus Mockford. (Roraima). Colombia.

133. D. pectenatus Eertmoed. (Bahia).

134. D. pictus Mockford. (Roraima).

135. D. pygmaeus (New). (Mato Grosso). 
136. D. similis Mockford. (Roraima).

137. D. spinosus Mockford. (Roraima).

138. Isthmopsocus barbatus Mockford. (Roraima).

139. I. breviceps (New). (Mato Grosso).

140. I. lanceatus Mockford. (Roraima).

141. I. luridus New. (Amazonas).

142. I. ornatus (New). (Mato Grosso).

143. I. radulatus New. (Bahia).

144. I. speculatus Mockford. (Roraima).

\section{Cladiopsocidae}

145. Cladiopsocus distinctus Eertmoed. (Paraná, Santa Catarina).

146. C. dolosus (Roesler). (Santa Catarina).

147. C. domesticus (New). (Mato Grosso).

148. C. fuscus (New). (Mato Grosso).

149. C. prionotus (New). (Amazonas).

150. C. uncinatus (New). (Amazonas).

\section{Ptiloneuridae}

151. Brasineura diamantina Silva Neto \& García Aldrete. (Bahia).

152. B. jiboia Silva Neto, García Aldrete \& Rafael. (Bahia).

153. B. serranortensis Silva Neto, García Aldrete \& Rafael. (Pará).

154. B. spinosa Silva Neto, García Aldrete \& Rafael. (Espirito Santo).

155. B. troglophilica Silva Neto \& García Aldrete. (Pará).

156. Euplocania ariasi Vinasco, González \& García Aldrete. (Goiás).

157. E. badonneli New \& Thornton. (Rondônia). Colombia. Peru.

158. E. bujariensis Silva Neto, García Aldrete \& Rafael. (Acre).

159. E. cearaense Silva Neto, García Aldrete \& Rafael. (Ceará).

160. E. cerata New. (Amazonas). Peru.

161. E. equorum Vinasco, González \& García Aldrete. (Pará).

162. E. hutchingsi Silva Neto, García Aldrete \& Rafael. (Acre).

163. E. manausensis Vinasco, González \& García Aldrete. (Amazonas).

164. E. marginata New \& Thornton. (Pará). Peru.

165. E. picta New. (Amazonas).

166. E. pseudopictaoides Silva Neto, García Aldrete \& Rafael. (Acre).

167. E. quinquedivisa Silva Neto, García Aldrete \& Rafael. (Amazonas).

168. E. rafaeli Vinasco, González \& García Aldrete. (Paraná).

169. E. tocantina Vinasco, González \& García Aldrete. (Pará).

170. E. uariniensis Silva Neto, García Aldrete \& Rafael. (Amazonas).

171. E. xavieri Silva Neto, García Aldrete \& Rafael. (Amazonas).

172. Loneura amazonica (New). (Amazonas).
173. Loneura baiana Lima, Silva-Neto, García Aldrete \& Bravo. (Bahia).

174. L. brasiliensis Roesler. (Santa Catarina).

175. L. maracaensis García Aldrete. (Roraima).

176. Ptiloneura baiana (Silva Neto, García Aldrete \& Rafael). (Bahia).

177. Ptiloneuropsis diamantina Silva Neto, García Aldrete \& Rafael. (Bahia).

178. P. immaculata Roesler. (Rio de Janeiro).

179. Timnewia greeni (New). (Mato Grosso). Ecuador.

180. T. jeaneae Silva-Neto, García Aldrete \& Rafael. (Roraima).

181. Triplocania ariasi New. (Amazonas, Bahia).

182. T. calcarata New. (Amazonas).

183. T. calori Silva Neto, García Aldrete \& Rafael. (Bahia).

184. T. capixaba Silva Neto, García Aldrete \& Rafael. (Espirito Santo).

185. T. caudata New. (Amazonas).

186. T. fapeam Silva Neto, García Aldrete \& Rafael. (Amazonas).

187. T. furcata New. (Mato Grosso). Colombia.

188. T. immaculata New. (Amazonas).

189. T. inpa Silva Neto, García Aldrete \& Rafael. (Amazonas).

190. T. lamasi Silva Neto, Rafael \& García Aldrete. (Mato Grosso). Colombia.

191. T. lamasoides Silva Neto, Rafael \& García Aldrete. (Rondônia). Colombia.

192. T. lunulata New. (Amazonas).

193. T. lucida Roesler. (Santa Catarina).

194. T. magnifica Roesler. (Santa Catarina, Paraná).

195. T. manauara Silva Neto, García Aldrete \& Rafael. (Amazonas).

196. T. manueli Silva Neto, García Aldrete \& Rafael. (Ceará).

197. T. mariaelinae Silva Neto, García Aldrete \& Rafael. (Pará).

198. T. mariateresae Silva Neto, Rafael \& García Aldrete. (Rio de Janeiro).

199. T. newi Silva Neto, Rafael \& García Aldrete. (Amazonas, Tocantins).

200. T. paranaensis Silva Neto, García Aldrete \& Rafael. (Paraná).

201. T. plaumanni Silva Neto, Rafael \& García Aldrete \& Rafael. (Santa Catarina).

202. T. reflexa Roesler. (Santa Catarina).

203. T. rondoniensis García Aldrete. (Rondônia).

204. T. rosae Silva Neto, García Aldrete \& Rafael. (Paraná, Minas Gerais).

205. T. trifida Silva Neto, Rafael \& García Aldrete. (Mato Grosso).

206. T. umbrata New. (Amazonas).

\section{Spurostigmatidae}

207. Spurostigma caatinga Silva Neto \& García Aldrete. (Bahia).

\section{Asiopsocidae}

208. Notiopsocus facilis Mockford. (Roraima). 
209. N. neotropicus (Machado Allison \& Papavero). (Mato Grosso).

210. N. simplex Banks. (Amazonas, Roraima).

211. Pronotiopsocus amazonicus Mockford. (Amazonas).

\section{Caeciliusidae}

212. Coryphaca inca Enderlein. (Amazonas, Santa Catarina). Argentina, Peru.

213. C. matona (New \& Thornton). (Mato Grosso).

214. Stenocaecilius antillanus (Banks). "Brazil". Widespread in the American tropics.

215. S. casarum (Badonnel). (Bahia). Widespread in the tropics.

216. Valenzuela adrianae (Mockford). (Roraima).

217. V. albofasciatus (Mockford). (Roraima).

218. V. albomarginatus (Enderlein). (Rio de Janeiro).

219. V. cinalus (New \& Thornton). (Mato Grosso).

220. V. claripennis (Mockford). (Roraima).

221. V. claristigma (New \& Thornton). (Mato Grosso, Roraima). Venezuela.

222. V. clayae (New \& Thornton). (Mato Grosso).

223. V. fasciatus (Enderlein). (Pará).

224. V. gemmatus (Mockford). (Roraima).

225. V. micans (New \& Thornton). (Mato Grosso, Pará, Roraima).

226. V. nigroticta (Williner). (Paraná).

227. V. obscuripennis (Mockford). (Roraima).

228. V. paradistinctus (New \& Thornton). (Mato Grosso).

229. V. posticoides (Mockford). (Roraima).

230. V. tuberculatus (New \& Thornton). (Mato Grosso, Roraima).

231. Enderleinella occidentalis Mockford. (Roraima).

232. Xanthocaecilius eroticus Mockford. (Santa Catarina).

233. X. granulosus Mockford. (Roraima). Mexico, Panama, Venezuela.

234. X. pallidus Mockford. (Roraima).

\section{Stenopsocidae}

235. Graphopsocus cruciatus (Linnaeus). (Pará, Rondônia). Holarctic. Probably introduced.

236. G. mexicanus Enderlein. (Paraná, Santa Catarina). Mexico, Venezuela.

\section{Amphipsocidae}

237. Dasypsocus nigrifrons (Roesler). (Santa Catarina). Venezuela.

238. D. roesleri (New \& Thornton). (Mato Grosso). Mexico, Venezuela.

239. Polypsocus coleopterus Roesler. (Santa Catarina).

240. P. delunatus Roesler. (Santa Catarina).

241. P. falcifer Roesler. (Santa Catarina).

242. P. fastosus Roesler. (Santa Catarina).

243. P. fuscopterus Mockford. (Roraima).

244. P. griseolineatus (Enderlein). (Amazonas). Peru.

245. P. lineatus Mockford. (Roraima).

246. P. ohausianus (Enderlein). (Roraima). Ecuador.
247. P. selenius Roesler. (Amazonas, Santa Catarina). Venezuela.

248. P. serpentinus Mockford. (Amazonas, Roraima).

249. P. suffuscus Roesler. (Santa Catarina).

250. P. unicolor Roesler. (Santa Catarina).

\section{Dasydemellidae}

251. Dasydemella gynopeza Roesler. (Santa Catarina).

252. D. setosa Roesler. (Santa Catarina).

\section{Lachesillidae}

253. Anomopsocus radiolosus (Roesler). (Mato Grosso). Costa Rica, Mexico.

254. Graphocaecilius interpretatus Roesler. (Paraná, Santa Catarina). Colombia.

255. Notolachesilla maracana Mockford. (Roraima).

256. Amazolachesilla ariasi García Aldrete \& Mockford. (Amazonas). Colombia.

257. Waoraniella vidali García Aldrete \& Mockford. (Amazonas).

258. Lachesilla acuminata Mockford. (Roraima).

259. L. aethiopica Enderlein. (Roraima). Tropical waif.

260. L. amarilla New. (Mato Grosso).

261. L. ariasi García Aldrete. (Amazonas). Bolivia.

262. L. bahiana García Aldrete. (Bahia).

263. L. belemensis García Aldrete. (Pará).

264. L. bicornata New \& Thornton. (Mato Grosso, Roraima).

265. L. bimaculata García Aldrete. (Pará). Belice, Colombia, Panama.

266. L. brasiliensis García Aldrete. (São Paulo).

267. L. braticagua García Aldrete. (Pará). Costa Rica, French Guiana.

268. L. breviforceps García Aldrete. (Santa Catarina).

269. L. capreola New. (Mato Grosso).

270. L. carioca García Aldrete. (Rio de Janeiro).

271. L. cladoclaspers García Aldrete \& Silva Neto. (Bahia).

272. L. columnaris García Aldrete. (Santa Catarina).

273. L. concava García Aldrete. (Rio Grande do Sul, Santa Catarina). Venezuela.

274. L. convexa García Aldrete. (Bahia). Panama.

275. L. cuala García Aldrete. (Pará, Paraná, Rio de Janeiro, Roraima, São Paulo). Mexico, Panama, Trinidad.

276. L. diamantina García Aldrete \& Silva Neto. (Bahia).

277. L. falcata García Aldrete. (Roraima, Sao Paulo). Bolivia, Colombia.

278. L. garcíai Mockford. (Roraima).

279. L. lapadoce García Aldrete \& Silva Neto. (Bahia).

280. L. marabaensis García Aldrete. (Pará).

281. L. marginata New \& Thornton. (Mato Grosso).

282. L. matogrossensis García Aldrete. (Mato Grosso).

283. L. megaforcepeta Mockford. (Roraima).

284. L. nevermanni (Navás). (Pará). Costa Rica, Honduras, Panama, Trinidad.

285. L. nilopecanhensis García Aldrete \& Silva Neto. (Bahia).

286. L. oriximinaensis García Aldrete \& Silva Neto. (Pará). 287. L. palmera New. (Mato Grosso, Goiás). 
288. L. papillata García Aldrete. (Pará). French Guiana.

289. L. patula García Aldrete. (Rondônia).

290. L. paulista García Aldrete. (Sao Paulo). Argentina.

291. L. pigmentithorax García Aldrete. (Amazonas). Colombia, Peru.

292. L. pilosiforceps García Aldrete. (Pará).

293. L. rugosa García Aldrete. (Rio de Janeiro, Santa Catarina, São Paulo). Colombia, Peru, Trinidad.

294. L. tectorum Badonnel. (São Paulo). Widely distributed.

295. L. teresiana García Aldrete. (Espírito Santo).

296. L. trunca García Aldrete. (Pará, Pernambuco). Panama.

297. L. valvula New \& Thornton. (Mato Grosso, Paraná). Colombia.

298. L. yanomami Mockford. (Pará, Roraima).

299. Nadleria alpha Badonnel \& García Aldrete. (Pará). Trinidad.

300. N. gamma Mockford. (Amazonas). Belice, Peru.

301. N. mockfordi Badonnel \& García Aldrete. (Pará).

\section{Ectopsocidae}

302. Ectopsocopsis cryptomeriae (Enderlein). (Rio Grande do Sul). Cosmopolitan.

303. Ectopsocus pumilis (Banks). (São Paulo). Widely distributed.

304. E. ribagai Enderlein. (Pará). Puerto Rico.

305. E. richardsi (Pearman). Brazil. Widely distributed.

306. E. titschacki Jentsch. (Bahia, Pará). Tropical waif.

307. E. vilhenai Badonnel. (Pará). Tropical waif.

\section{Peripsocidae}

308. Peripsocus australis Mockford. (Santa Catarina).

309. P. nubifer Mockford. (Roraima).

310. P. pauliani Badonnel. "Brazil". Widely distributed.

311. P. phacellodomi New. (Pernambuco).

312. P. placidus Mockford. (Roraima).

313. P. subtristis Mockford. (Roraima).

314. P. teutonicus Mockford. (Santa Catarina).

315. P. tristis Mockford. (Roraima).

\section{Archipsocidae}

316. Archipsocopsis frater (Mockford). (Sao Paulo). EUA, Jamaica, Mexico.

317. A. inornata New. (Mato Grosso, Sao Paulo). Mexico, Panama, Surinam, Trinidad, Venezuela.

318. A. virgata New. (Pará, Roraima). French Guiana, Surinam, Venezuela.

319. Archipsocus badonneli New. (São Paulo). Colombia, Venezuela.

320. A. brasilianus Enderlein. (Pará). Paraguay, Puerto Rico.

321. A. broadheadi Badonnel. (Pará). Panama.

322. A. castrii Badonnel. (Sao Paulo). Colombia.

323. A. cervinus New. (Pará, Rio de Janeiro). Colombia, Surinam, Venezuela.

324. A. costalimai New. (Pará, São Paulo).
325. A. enderleini New. (Mato Grosso).

326. A. floridanus Mockford. (São Paulo). Florida, Mexico.

327. A. gibberophallus New. (Mato Grosso). French Guiana.

328. A. granulosus Badonnel. (Mato Grosso).

329. A. gurneyi Mockford. (Roraima). EUA, Jamaica, Mexico.

330. A. indentatus Mockford. (Roraima).

331. A. lenkoi Badonnel. (Roraima).

332. A. lineatus New. (Pará).

333. A. minutillus New. (Amazonas, Mato Grosso).

334. A. mockfordi New. (Mato Grosso, Pará, Roraima, São Paulo). Panama, Peru, Surinam, Trinidad, Venezuela.

335. A. modestus New. (Mato Grosso). Colombia.

336. A. newi Badonnel. (São Paulo).

337. A. pearmani New. (Mato Grosso). Panama.

338. A. tenebricosus New. (Mato Grosso).

339. Notarchipsocus fasciipennis Mockford. (Roraima).

340. N. macrurus (New). (Mato Grosso, Roraima) Panama, Trinidad.

\section{Pseudocaeciliidae}

341. Pseudocaecilius tahitiensis (Karny). (Pará, Rio de Janeiro, Roraima). Widespread in the tropics.

342. Scytopsocus coriaceus Roesler. (Santa Catarina). Cuba, Jamaica, Mexico.

343. S. difficilis Roesler. (Santa Catarina).

344. S. fluminis Mockford. (Roraima).

345. S. medialis New \& Thornton. (Mato Grosso).

\section{Philotarsidae}

346. Haplophallus leopardina (Williner). (Paraná). Argentina, Colombia.

347. Aaroniella bruchi (Williner). (Roraima). Argentina.

348. A. glabra New \& Thornton. (Santa Catarina).

349. A. recta New \& Thornton. (Mato Grosso).

\section{Elipsocidae}

350. Nepiomorpha brasiliana Badonnel. (São Paulo). Mexico, Venezuela.

351. N. cingulata New. (Mato Grosso).

352. N. dolosa Badonnel. (São Paulo).

353. N. pallida New. (Mato Grosso, Roraima).

\section{Hemipsocidae}

354. Hemipsocus pallidus New \& Thornton. (Mato Grosso).

\section{Psocidae}

355. Blaste alfineta New. (Mato Grosso).

356. B. amazonica García Aldrete. (Roraima).

357. B. caudata Mockford. (Roraima).

358. B. forcepata New. (Mato Grosso).

359. B. fusimera New \& Thornton. (Paraná).

360. B. hamata Mockford. (Roraima).

361. B. ligula New \& Thornton. (Paraná). Uruguay.

362. B. longispina Mockford. (Roraima).

363. B. macrura (New). (Amazonas, Mato Grosso, Roraima). 
364. B. obscura (New). (Mato Grosso).

365. B. plaumanni New. (Paraná).

366. B. richardsi New. (Paraná).

367. Blastopsocidus brasiliensis (New). (Mato Grosso).

368. Blastopsocus mockfordi Badonnel. (Roraima). Colombia.

369. Elaphopsocus amazonicus Román, González \& García Aldrete. (Amazonas).

370. E. bahianus Román, González \& García Aldrete. (Bahia).

371. E. glaphyrostigma Roesler. (Santa Catarina).

372. Cerastipsocus beaveri New. (Mato Grosso, Paraná).

373. C. brasilianus (Enderlein). (Espírito Santo).

374. C. consocius (Navás). "Brazil”.

375. C. fuscipennis (Burmeister). "Brazil".

376. C. kolbei New. (Mato Grosso).

377. C. rugosus New \& Thornton. (Paraná).

378. C. sivorii (Ribaga). (São Paulo). Uruguay.

379. C. willineri New \& Thornton. (Paraná).

380. Cervopsocus medialis New. (Amazonas, Pará, Rondônia). Belice, Colombia, Peru.

381. Dactylopsocus fumigatus (Kolbe). (Santa Catarina).

382. D. similis Mockford. (Roraima).

383. Eremopsocus crassicornis (Kolbe). (Minas Gerais, Santa Catarina). Argentina, Uruguay.

384. E. infumatus McLachlan. (Goiás). Venezuela.

385. E. nigripes Mockford. (Santa Catarina).

386. Psococerastis amazonica New. (Amazonas).

387. P. callangana (Enderlein). (Amazonas Espírito Santo, Minas Gerais, Pernambuco, Rio de Janeiro, Santa Catarina). Panama, Peru.

388. P. fasciata Mockford. (Amazonas, Espírito Santo, Pará, Rio de Janeiro, Santa Catarina). Belice, Mexico, Panama.

389. P. hageni New. (Mato Grosso).

390. P. opulenta (Navás). (Amazonas, Goiás, Mato Grosso, Pará, Pernambuco, Rio de Janeiro, São Paulo).

Widely distributed in tropical America.

391. P. paraguayana (Ribaga). (Santa Catarina, São Paulo). Paraguay.

392. P. pictiventris (Kolbe). (Santa Catarina).

393. P. pyralina (Kolbe). (Minas Gerais, Santa Catarina).

394. P. stictica (Banks). "Brazil”.

395. Brachinodiscus cinctipes (Enderlein). "Brazil". Bolivia, Paraguay, Peru.

396. Metylophorus bishopi New. (Mato Grosso).

397. M. calcaratus Mockford. (Roraima).

398. M. ctenatus New. (Mato Grosso).

399. M. fasciatus New. (Amazonas).

400. M. hispidus Mockford. (Roraima).

401. M. symmetricus Mockford. (Roraima).

402. M. theresopolitanus (Enderlein). (Rio de Janeiro).

403. Opthalmopsocus ocularis (Kolbe). "Brazil".

404. O. pallidus New. (Mato Grosso, Roraima).

405. Indiopsocus paranensis (New \& Thornton). (Paraná).

406. I. sinuatistigma (New). (Mato Grosso).

407. Psocidus albostigmus (Banks). "Brazil".

408. P. manausensis New. (Amazonas).

409. P. fuscatus (Navás). "Brazil”.

410. P. kotzbaueri (Navás). "Brazil".
411. P. nigricornis (Brauer). "Brazil".

412. P. proi (Navás). (Rio Grande do Sul). Argentina.

413. P. punctaticeps (Enderlein). "Brazil".

414. P. quadrisignatus (Banks). (Amazonas).

415. P. simplex (Enderlein). "Brazil".

416. P. zikani (Navás). "Brazil".

417. Psocomesites continuatus (Roesler). (Pará).

418. Ptycta lunulata New. (Mato Grosso).

419. P. pearmani New. (Mato Grosso).

420. P. reticulata New. (Mato Grosso).

421. Steleops albertonetoi González, García Aldrete \& Carrejo. (Bahia).

422. S. maculatus New. (Mato Grosso).

423. S. pedunculatus (Enderlein). Brazil. Paraguay, Peru.

424. S. pulcher New. (Mato Grosso).

425. Trichadenotecnum circularoides Badonnel.

(Roraima). Widely distributed.

426. T. gonzalezi (Williner). (Roraima). Argentina.

427. T. pardus Badonnel. (Pará). Widely distributed.

428. T. roesleri New. (Mato Grosso).

429. T. shawi Yoshizawa \& García Aldrete. (Goiás).

430. T. simile Mockford. (Roraima).

431. T. sinuatum New. (Mato Grosso).

432. T. ufla Yoshizawa. (Minas Gerais).

433. Dictyopsocus pennicornis (Burmeister). (Amazonas, Bahia, Ceará, Paraíba, Santa Catarina). Argentina, Venezuela.

434. Poecilopsocus anticus (Walker). (Pará).

435. P. beaveri (New). (Mato Grosso).

436. P. bishopi (New). (Mato Grosso).

437. P. cinctus (Enderlein). (Amazonas).

438. P. leucotelus (Walker). (Pará).

439. P. richardsi (New). (Mato Grosso).

440. Thyrsophorus formosus Navás. "Brazil".

441. T. speciosus Burmeister. (Pará). French Guiana, Panama, Peru, Surinam.

442. Thyrsopsocus aequatorialis Enderlein. "Brazil". Ecuador.

443. T. bellus (McLachlan). (Pará). Bolivia, Peru.

444. T. brasiliensis New. (Amazonas).

445. T. elegans (Enderlein). Brazil.

446. Thyrsosocopsis amazonicus Román, García Aldrete \& González. (Amazonas).

\section{Myopsocidae}

447. Lichenomima ariasi (New). (Amazonas, Roraima). 448. L. conspersa Enderlein. (Espírito Santo). Paraguay. 449. L. onca Mockford. (Roraima). Colombia.

450. L. pulchella (New \& Thornton). (Mato Grosso).

451. L. sparsa (Hagen). (Espírito Santo). Canada, Cuba, Mexico, USA.

452. L. thorntoni Mockford. (Roraima).

453. L. timmei Mockford. (Roraima).

454. Myopsocus albomaculatus (New). (Amazonas).

455. M. albus García Aldrete. (Roraima).

456. M. cinctus (New). (Amazonas).

457. M. minor (New \& Thornton). (Mato Grosso). Colombia.

458. M. parvus Mockford. (Roraima).

459. M. pennyi (New). (Amazonas).

460. M. vespertilio Mockford. (Roraima). 


\section{Key to families of Psocoptera from Brazil}

\section{Adapted from García Aldrete \& Mockford (2012) and García Aldrete et al., 2018.}

1. Adults with more than 18 flagellomeres; hypopharyngeal filaments separate in their entire length, never fused on midline; labial palpus with minute basal segment and rounded distal segment. Suborder Trogiomorpha ... 3

- Adults with less than 18 flagellomeres (usually 11-13); hypopharyngeal filaments fused along midline at least for part of their length; labial palpus as above, or only one-segmented..

2. Adults with 13 flagellomeres (rarely less); at least some flagellomeres annulated with cuticular sculpture. Labial palpus usually with a minute basal segment and a rounded distal segment. Tarsi usually three-segmented. Forewing, when present, lacking sclerotized pterostigma............ Suborder Troctomorpha ... 7

- Adults with 11 flagellomeres (rarely less); no flagellomeres annulated with cuticular sculpture but sometimes with reticulate sculpture in cuticle. Labial palpus lacking basal segment, consisting of a single rounded or triangular segment. Tarsi two-or three-segmented. Forewings, when fully developed, with sclerotized pterostigma Suborder Psocomorpha ... 11

3. Lacinia slightly simplified. Paraprocts lacking anal spine.. Infraorder Prionoglaridetae: Prionoglarididae

- Lacinia normally developed. Paraprocts with anal spine .... 4

4. Forewings, when fully developed, with vein CU2 (CuP) and IA ending separately on wing margin; spur sensillum always present on the second segment of maxillary palpus (Mx2). Ovipositor valvulae: v3 elongate, partially joined together on midline by membrane; v2 small or absent; v1 absent.

Infraorder Atropetae ... 5

— Forewings, when fully developed, with veins Cu2 (CuP) and IA ending together on wing margin (point of junction called 'nodulus'); spur sensillum of Mx2 present or absent. Ovipositor valvulae: 33 never elongate, but sometimes very broad; v2 usually present, slender; v1 frequently present, slender.

Infraorder Psyllipsocetae: Psyllipsocidae

5. Body and forewings covered with scales or dense setae. Wings often pointed apically, with visible veins. Mesocoxae of the two sides capable of interlocking by a'button and cavity' mechanism. Lepidopsocidae

— Body and forewings never covered with scales or dense setae. Wings not pointed apically, with visible veins. Mesocoxae of the two sides not capable of interlocking...

6. Wings, even when very small, with distinct, visible veins. Ovipositor only with valve 3. Opening of the the spermateca with two conspicuous accessory bodies... Psoquillidae

— Wings reduced, without distinct veins, occasionally absent. Ovipositor mainly with valve 3 , but usually also with valve 2 . Two denticulated accessory plates (maculas) attached to spermateca. .Trogiidae

7. Small forms, rarely over $2 \mathrm{~mm}$ in length. Males wingless, females usually wingless or brachypterous. Forewings of winged females with not more than two $\mathrm{M}$ branches and lacking vein A2. Pearman's coxal organ absent or represented by a slight bulge in cuticle ...nfraorder Nanopsocetae ... 8

- Body size variable but usually at least $3 \mathrm{~mm}$ in length. Wings present in adults of both sexes, forewings with $M$ three-branched and vein IIA usually present. Pearman's coxal organ represented by a mirror and a rasp. Infraorder Amphientometae ... 9

8. Body flattened; coxae of opposite sides widely separated by broad sternal plates. Forewings, when present (some females) with two parallel longitudinal veins occupying main body of wing..... Liposcelididae

— Body not flattened; coxae of opposite sides only narrowly separated. Forewings, when present (some females), with several branching veins occupying main body of wing.

9. Body and forewings densely covered with scales. In forewing veins $A 1$ and $A 2$ separate for their entire length...

- Body and forewings lacking scales. In forewing veins IA and IIA joined together before reaching wing margin Pachytroctidae

10. Forewing with a long Rs-M crossvein; forewings unpigmented. Outer cusp of lacinial tip with only two denticles Amphientomoidea: Amphientomidae

— Rs and M in forewing joined at a point; forewings with cloudy pigmentation throughout or with distinct spotting and banding pattern. Outer cusp of lacinial tip with three denticles. Troctopsocidae

11. Meso-precoxal bridges narrow and corresponding mesotrochantins broad basally. Labrum curved on sides, well molded to contours of mandibles.

Infraorder Psocetae . 32

- Meso-precoxal bridges and mesotrochantins not as above (i.e., the former wide and the latter narrow basally), or, if otherwise, labrum broad and flat, bearing a longitudinal pair of sclerites. Infraorder Caeciliusetae ... 18

12. Pretarsal claw lacking preapical denticle, forewings and body not densely-setose.

- Pretarsal claw usually with a preapical denticle; if denticle absent (Family Archipsocidae) forewings and body densely-setose... 13

13. V3 largely fused to V2. Labrum with a pair of longitudinal sutures running through it. Labrum usually flat, not conforming closely to contours of mandibles; mandibles elongate

Infraorder Epipsocetae ... 14

— V3 largely free from V2 and from body wall. Labrum curved on sides, well molded to contours of mandibles, lacking a pair of longitudinal sutures running through it.. ...nfraorder Homilopsocidea ... 22

14. Forewings with one anal vein Epipsocidae

- Forewings with two anal veins 15

15. Forewings with length of $A 2$ equal to or greater than half the length of $A 1$; pterostigma with a spur-vein Spurostigmatidae

- Forewings with length of A2 less than half the length of A1; pterostigma without a spur-vein (except Timnewia)

16. Lacinial tip with outer cusp slender, bearing not more than three denticles. Pretarsal claw with pulvillus bent near base, expanded at tip...... Dolabellopsocidae

- Lacinial tip with outer cusp broader, bearing more than three denticles. Pretarsal claw with pulvillus straight and pointed distally

17. Pretarsal claw with a basal spine in addition to the more distal pulvillus. Forewings with or without spur veins, $M$ with three branches. The two labral sutures reaching proximal margin of labrum and curving around to lateral margin 
— Pretarsal claw with only the spine-like pulvillus. Forewing veins lacking spur veins, $M$ with three to nine branches. The two labral sutures never reaching proximal margin of labrum, except in Willreevesia ....

Ptiloneuridae

18. Ventral abdominal vesicles absent. Mesepistemal sulcus absent. Mandibles short to moderate in length..................... Superfamily Asiopsocoidea: Asiopsocidae

— With one to three ventral abdominal vesicles. Mesepisternal sulcus present. Mandibles elongate ........................................... Superfamily Caeciliusoidea ... 19

19. Forewings with a crossvein from the pterostigma to Rs stem and another from the $\mathrm{M}$ stem to areola postiça...................................................... Stenopsocidae

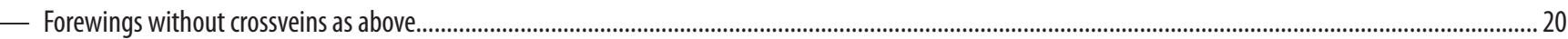

20. Ciliation of hindwing margin restricted to cell $\mathrm{r} 3$ or none. Mx4 longer than $M \times 2$. Spine of free margin of paraproct large .................................. Dasydemellidae

- Ciliation of hindwing margin complete except for basal two-thirds of front margin. Mx4 shorter than or equal to Mx2. Spine of free margin of paraproct small or absent

21. Setae of veins in distal half of forewing on both dorsal and ventral surfaces, vein $M 2$ or 3-branched. $M x 4=M \times 2$ in length ............................... Amphipsocidae
- Setae of veins in distal half of forewing only on dorsal surface, vein $M$ 3-branched. $M x 4$ shorter than $M x 2$..................................................eciliusidae

22. In forewings, setae of wing margin in $\mathrm{m}$ cells in two series forming crossing pairs. Brachypterous and micropterous individuals with numerous long, backwarddirected setae on vertex and thoracic dorsum.

- Forewing margin lacking series of crossing hairs. Brachypterous and micropterous individuals with at most only moderate body ciliation .............................. 25

23. Venation in forewing vague; Rs in forewing often unbranched. Wings often greatly reduced; in both macropterous and short-winged forms numerous long, backward directed setae on vertex and thoracic dorsum. Usually colonial forms living under dense webbing................................................... Archipsocidae

- Venation in forewing distinct; Rs in forewing with two branches. Wings usually not reduced. Vertex and thoracic dorsum lacking numerous long, backwarddirected setae. Generally solitary, or a few individuals living together, either in open or under light webbing

24. Adults with tarsi two-segmented. External parameres generally much longer than aedeagal arch. Leaf-inhabiting forms .............................. Pseudocaeciliidae

- Adults usually with three tarsal segments. External parameres only slightly longer or shorter than aedeagal arch. Bark-inhabiting forms...............Philotarsidae

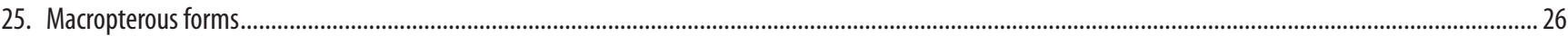

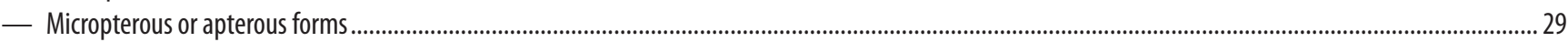

26. Forewings with vein CuA1 present and usually free from M. Wings either unciliated or lightly ciliated. Ovipositor reduced to one (v3), rarely two valvulae on each side.

Lachesillidae

— Forewings lacking vein CuA1. Wings lightly to moderately ciliated. Ovipositor usually with three valvulae, rarely reduced to one on each side......................... 27

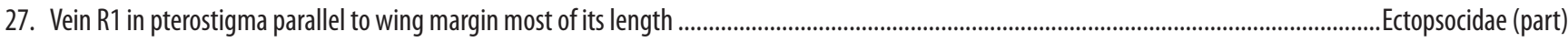

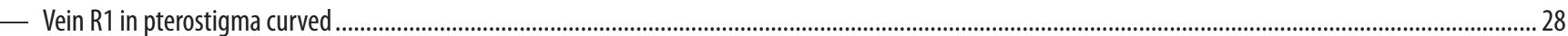

28. Both sexes macropterous. Phallosome elongate, with heavily sclerotized endophallic structure distally in form of a three-pronged fork........ Peripsocidae (part)

— Either males or females macropterous, not both. Endophallus not as above..................................................................................................

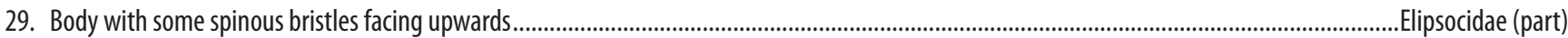

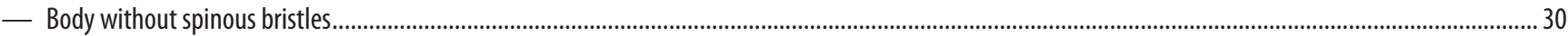

30. Antenna much shorter than the body. Subgenital plate with a distal median lobe, simple, thin (egg guide) ................................................... lipsocidae (part)

— Antenna about as long as the body. Subgenital plate with a relatively wide median distal protuberance or with two distal protuberances ............................... 31

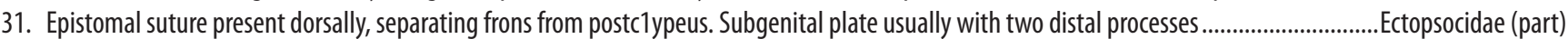

- Epistomal suture absent dorsally, postclypeus and frons continuous. Subgenital plate with a single, relatively broad median distal process....Peripsocidae (part)

32. Vein $M$ in forewing two-branched, the $M$ stem joined to vein CuA1 by a long crossvein; hind tibia lacking longitudinal row of ctenidiobothria...... Hemipsocidae

- Vein $\mathrm{M}$ in forewing three-branched, the $\mathrm{M}$ stem usually fused for a short distance to vein CuA1; rarely the two joined by a short crossvein. Hind tibia with a longitudinal row of ctenidiobothria

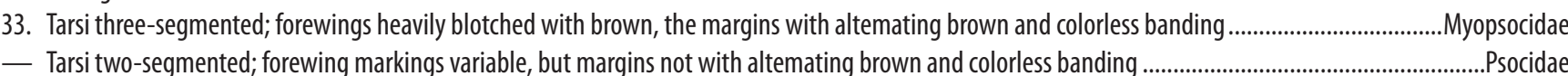

\section{DISCUSSION}

García Aldrete \& Mockford (2009) listed 425 species, in 94 genera and 28 families of Psocoptera for Brazil; thirty-nine species not described and a new genus not described (Genus ca. Goja) were included in this list. Based on the data above, there were 385 species and 93 genera described or recorded for Brazil to 2009. In this paper, only described species were considered, totaling 460 species in 97 genera and 30 families for the Brazilian psocid fauna (Figs. 1, 2). The number of psocid species recorded in Brazil was increased from 2009 to the beginning of 2020 by seventy-six species, four genera (Lithoseopsis Mockford, Neotrogla Lienhard, Ptiloneura Enderlein and Spurostigma Eertmoed) and two families (Prionoglarididae Karny and Spurostigmatidae Eertmoed).

Lienhard \& Smithers (2002) recorded for South America 762 species and also suggested that the num- ber of species recorded in Brazil, and in general for South America was highly underestimated. Rafael et al. (2012) in the preface of the book "Insects from Brazil" estimated a total of 2.000 psocid species for Brazil. If this estimation made is close to reality, there would still be around 1.500 psocid species to be described or recorded for Brazil, and hence, these numbers suggest that we still do not know $75 \%$ of the Brazilian psocid fauna.

In 2018 a list of 720 species of Psocoptera recorded in Colombia (including species not described) was published and the number of Colombian psocids was therefore increased by $811.4 \%$ (García Aldrete et al., 2018). In the list of Colombian species, out of the 720 listed species, only 320 were described species, and therefore Brazil is the country with the most diverse known psocid fauna of South America. Silva-Neto et al. (2014b) recorded 175 undescribed psocid species for the Northeast region of Brazil. In addition, direct observation made by 
one of us (AMSN) confirm dozens of other undescribed species stored and identified at the level of genus in several Brazilian collections, especially in the collection of the Instituto Nacional de Pesquisas da Amazônia (INPA), in Manaus, Amazonas; the Entomological Collection Prof. Johann Becker of the Zoology Museum of the Universidade Estadual de Feira de Santana, in Feira de Santana, Bahia, Brazil (MZFS) and the Invertebrate
Collection of the Biology Department, Universidade Federal de Lavras, Minas Gerais, Brazil.

The largest and most diverse Brazilian psocid family is Psocidae, with 92 species in 23 genera, followed by Ptiloneuridae (56 species in 7 genera), Epipsocidae (46 species in 5 genera), and Lachesillidae (49 species in 7 genera) (Figs. 1, 2). Ptiloneuridae was the family that most increased its diversity in Brazil, from 19 species and

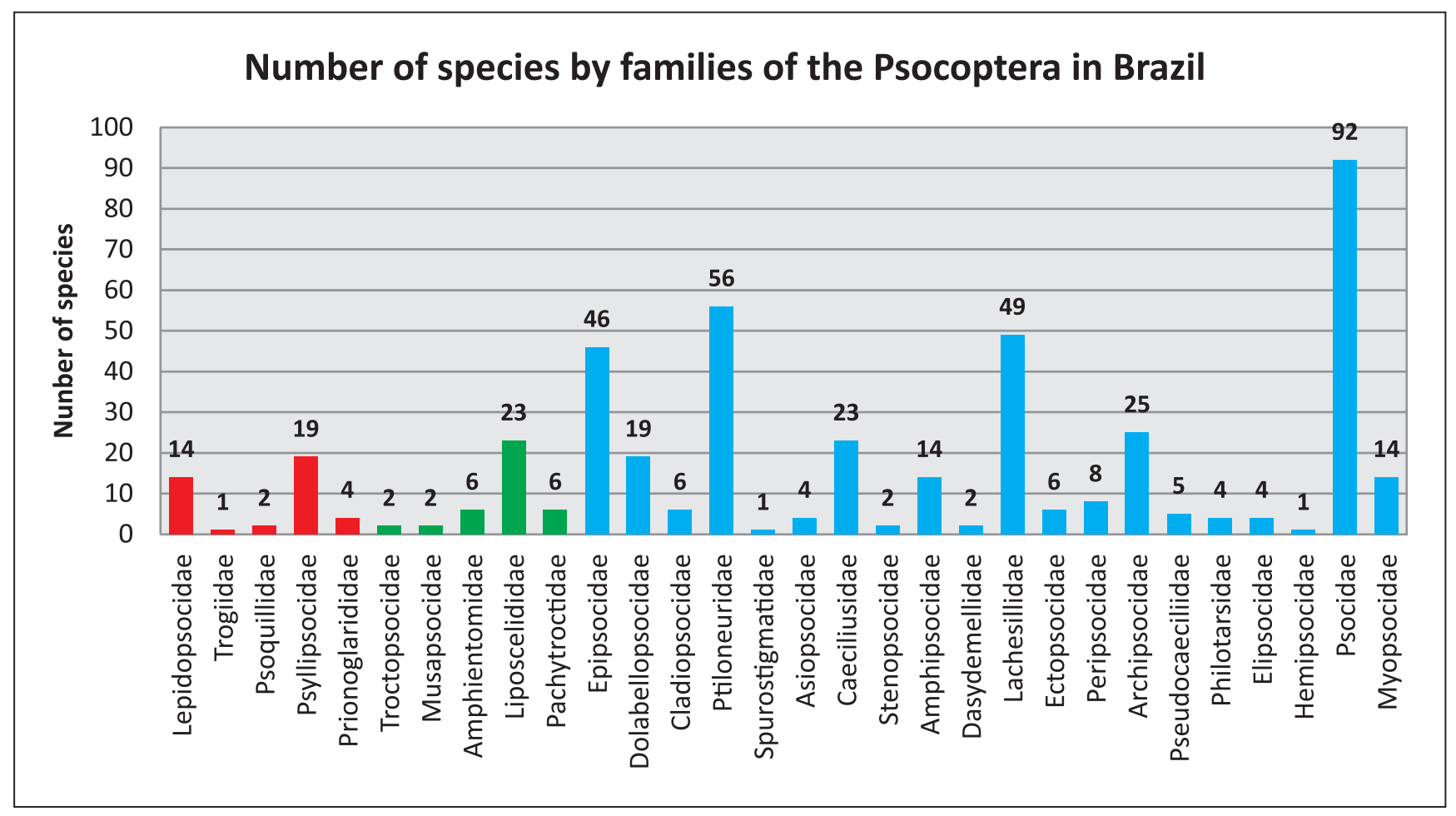

Figure 1. Number of Brazilian psocid species by family. Red Trogiomorpha families. Green Troctomorpha families. Blue Psocomorpha families.

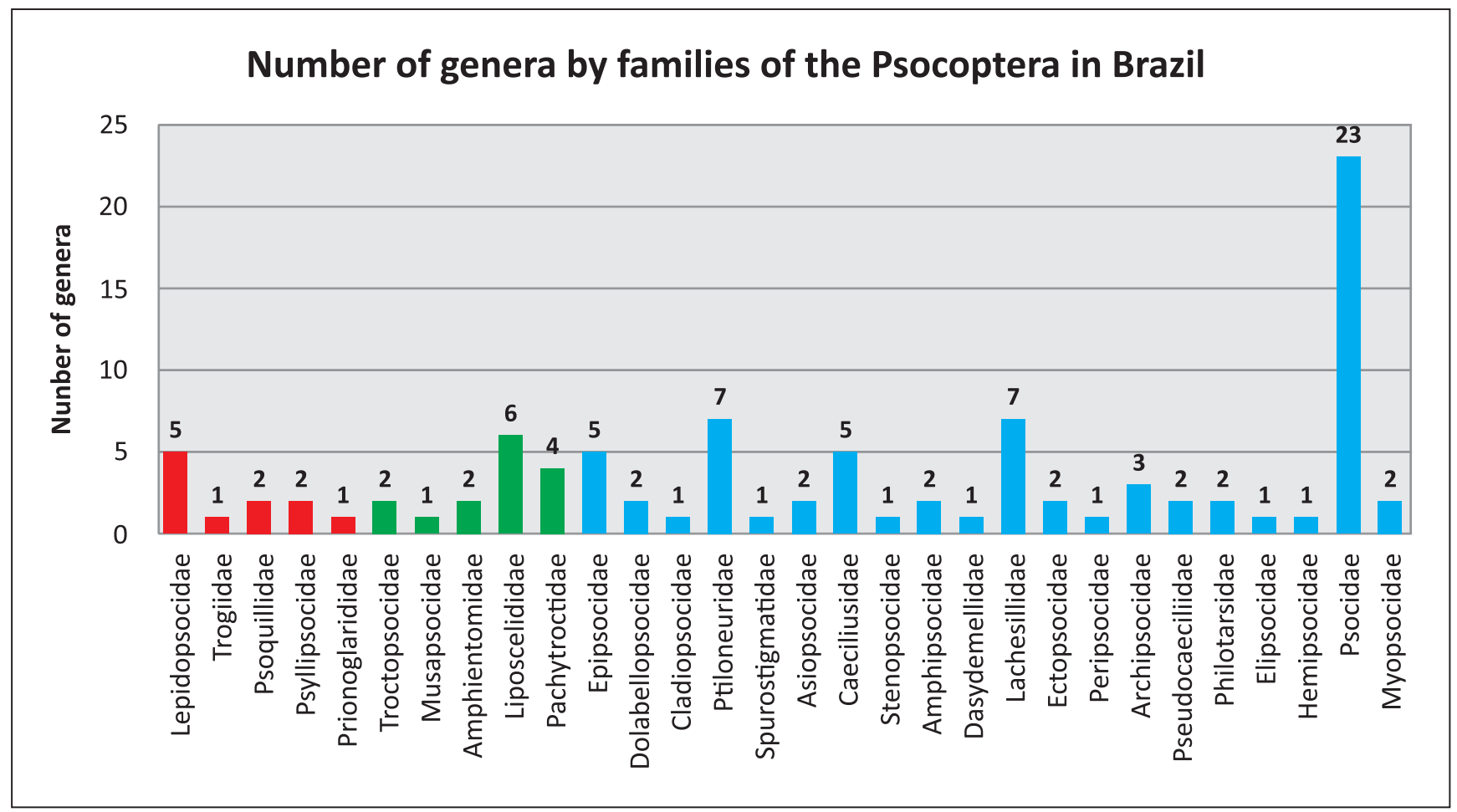

Figure 2. Number of Brazilian psocid genera by family. Red Trogiomorpha families. Green Troctomorpha families. Blue Psocomorpha families. 
five genera in 2009 to 56 species and seven genera in 2020 (Figs. 1, 2).

Brazil is divided in 26 states and a Federal District. Psocid species were recorded in 23 of these Brazilian states (Fig. 3). The northern region of Brazil is the most diverse in number of species, with 228 species, totaling $49 \%$ of all the Brazilian psocid fauna and especially the following states: Roraima (91 species), Amazonas (77 species) and Pará (50 species) (Fig. 3).

Among the Brazilian regions, the Northeast region has the lowest number of Psocoptera species records (Fig. 3). This fact has already been emphasized by SilvaNeto et al. (2014a), but the number of psocids species recorded in this Brazilian region has increased considerably, from 22 species in 2009 to 58 species in 2020. The increase of $263 \%$ in the number of Psocoptera fauna in the Northeast region is due to the study of fauna in the state of Bahia (fauna inventories of the program PPBioSemi-Árido, Universidade Estadual de Feira de Santana) and studies of cave fauna in northeastern states (The program Cave invertebrates in Brazil, Universidade Federal de Lavras).

The most diverse Brazilian state in terms of number of families, genera and species is the Brazilian state of Mato Grosso, located in the Midwest region of Brazil (Fig. 3). Mato Grosso had its South region separated in 1977 to originate the state of Mato Grosso do Sul. The most species of Psocoptera described for that location were collected before 1977 and had information not so accurate on exact collection locations, sometimes just information: Mato Grosso. In this way, many of the species in these papers recorded in Mato Grosso, may have been

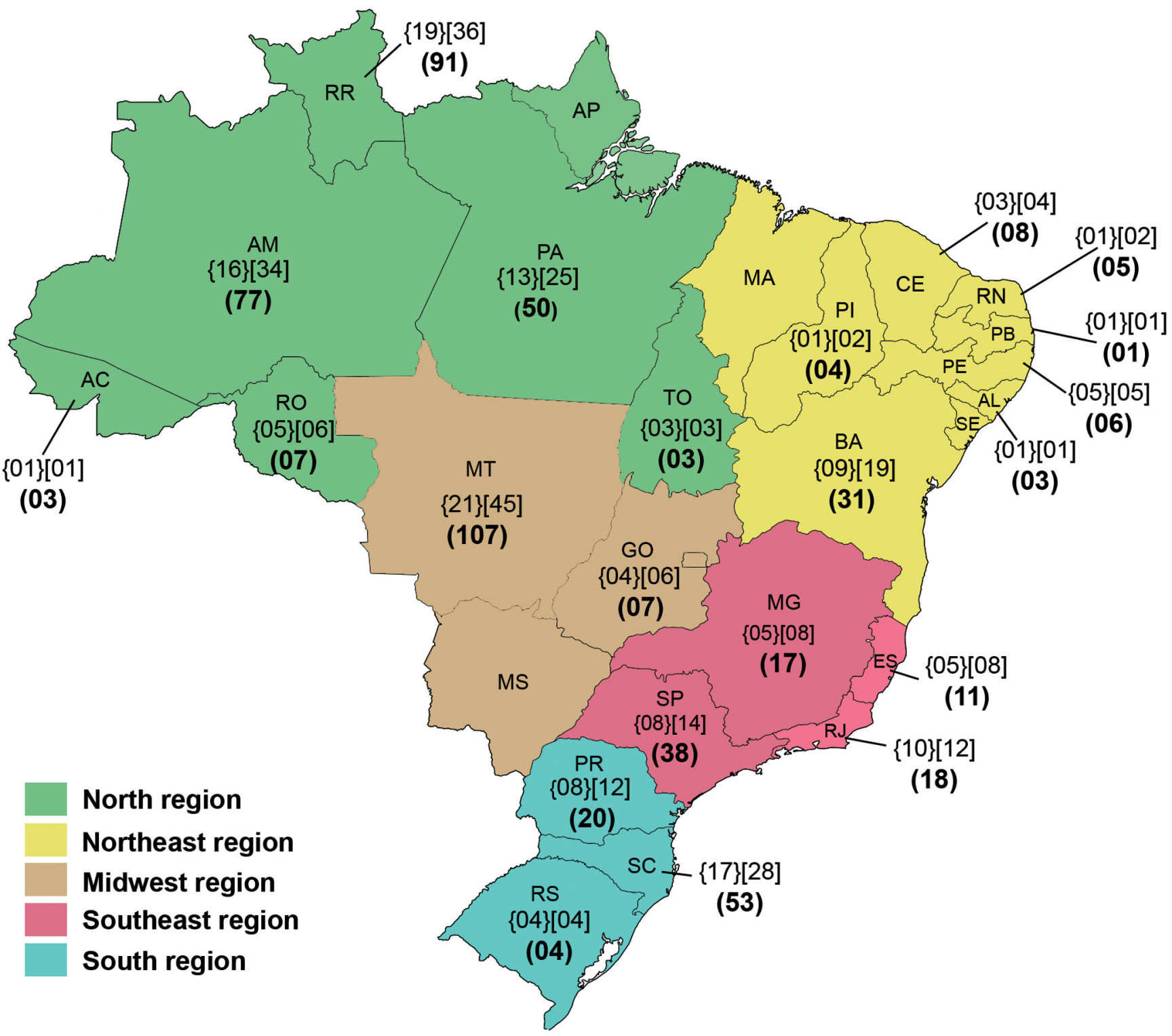

Figure 3. Distribution map of psocid families, genera and species by Brazilian states. Families between curly brackets, genera between square brackets and species between parentheses. The species number sum in the map does not represent the number of recorded species in Brazil, as one species may have been recorded in more than one State. Abbreviations of the Brazilian states: $\mathrm{AC}=\mathrm{Acre}, \mathrm{AM}=$ Amazonas, $\mathrm{RR}=$ Roraima, $\mathrm{RO}=$ Rondônia, $\mathrm{AP}=\mathrm{Amapá}, \mathrm{PA}=\mathrm{Para}, \mathrm{TO}=\mathrm{Tocantins}, \mathrm{MT}=\mathrm{Mat0}$ Grosso, MS = Mato Grosso do Sul, $\mathrm{GO}=$ Goiás, $\mathrm{MA}=$ Maranhão, $\mathrm{PI}=$ Piauí, $\mathrm{CE}=$ Ceará, $\mathrm{RN}=$ Rio Grande do Norte, $\mathrm{PB}=$ Paraíba, $\mathrm{PE}=\mathrm{Pernambuco}, \mathrm{AL}=\mathrm{Alagoas}$, $\mathrm{SE}=$ Sergipe, $\mathrm{BA}=$ Bahia, MG = Minas Gerais, ES = Espírito Santo, RJ = Rio de Janeiro, SP = São Paulo, PR = Paraná, SC = Santa Catarina e RS = Rio Grande do Sul. 
collected in the current region of the Mato Grosso do Sul and perhaps for this reason, the latter is one of the three Brazilian states without records of Psocoptera species.

In the southern region of Brazil, the state of Santa Catarina appears with a considerable diversity with 53 species recorded. Most of the psocids species in the Brazilian state of Santa Catarina were collected and sent to foreign Psocoptera specialists, by the German entomologist Fritz Plaumann, resident in Santa Catarina, who in 70 years of work (1924 to 1994), catalogued about 80 thousand specimens of 17 thousand different insect species, of which 1,500 were unknown to science and among these hundreds of psocid species.

In addition to the megadiversity of the Brazilian psocid fauna, its high level of endemism is remarkable, with $75 \%$ (343) of Brazilian psocid species exclusive to its na- tional territory. In the future, with the increase of studies of psocid fauna in South America, many of the species endemic to Brazil may lose that status. For example, with the publication of the Colombian psocid fauna (García Aldrete et al., 2018), twelve species (Epipsocus badonneli Mockford, Mesepipsocus brasiliensis (New), Neurostigma xanthopterum New, Dolabellopsocus lobatus Mockford, Triplocania lamasi Silva Neto, Rafael \& García Aldrete, Triplocania lamasoides Silva Neto, Rafael \& García Aldrete, Triplocania furcata New, Graphocaecilius interpretatus Roesler, Amazolachesilla ariasi García Aldrete \& Mockford, Lachesilla valvula New \& Thornton, Lichenomima onca Mockford and Myopsocus minor (New \& Thornton)) that were considered endemic to Brazil in the list of García Aldrete \& Mockford (2009) are no longer exclusive to the Brazilian fauna.

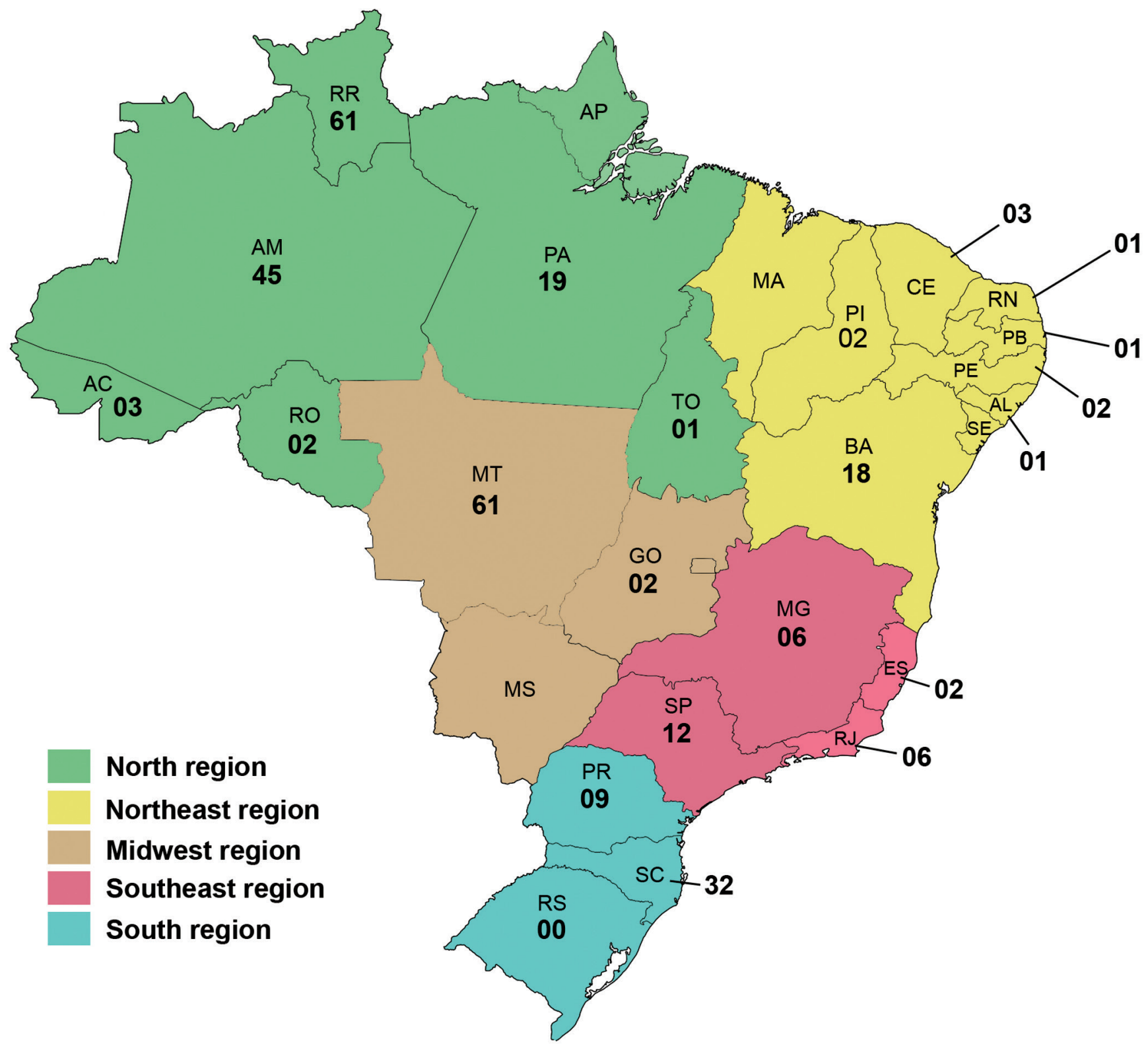

Figure 4. Distribution map of endemic psocid species by Brazilian states. Abbreviations of the Brazilian states: $A C=A c r e, A M=A m a z o n a s, R R=R o r a i m a, R 0=$ Rondônia, $\mathrm{AP}=$ Amapá, $\mathrm{PA}=$ Pará, $\mathrm{TO}=$ Tocantins, $\mathrm{MT}=$ Mato Grosso, MS = Mato Grosso do Sul, $\mathrm{GO}=$ Goiás, MA = Maranhão, $\mathrm{PI}=$ Piauí, $\mathrm{CE}=\mathrm{Ceará}, \mathrm{RN}=\mathrm{Rio}$ Grande do Norte, $\mathrm{PB}=$ Paraíba, $\mathrm{PE}=$ Pernambuco, $\mathrm{AL}=$ Alagoas, $\mathrm{SE}=$ Sergipe, $\mathrm{BA}=$ Bahia, MG = Minas Gerais, ES = Espírito Santo, RJ = Rio de Janeiro, $\mathrm{SP}=\mathrm{São}$ Paulo, PR = Paraná, $S C=$ Santa Catarina e RS = Rio Grande do Sul. 
The Brazilian state with the largest number of endemic psocid species is Mato Grosso (61 species), followed by the states of Roraima (61 species), Amazonas (45 species), Santa Catarina (32 species) and Bahia 18 (species) (Fig. 4). In the future, with the increase of studies of the psocid fauna in different states and regions, the tendency is that cases of endemism by states and regions will have a considerable decrease, demonstrating more clearly the true patterns of distribution of species of Psocoptera in Brazil.

\section{ACKNOWLEDGEMENTS}

AMSN thanks Instituto Nacional de Pesquisas da Amazônia (INPA) and Coordenação de Aperfeiçoamento de Pessoal de Nível Superior (CAPES) for research support. A.M.S.N. thanks particularly the support for the Capes-INPA research grant (Process: 88887.312051/2018-00). ANGA thanks Instituto de Biología, Universidad Nacional Autónoma de México, for continuous research support.

\section{REFERENCES}

Alves-Oliveira, J.; Silva-Neto, A.M.; Mendes, D.M.M. \& García Aldrete, A.N. 2017. New records of Dictyopsocus pennicornis (Burmeister) (Psocodea 'Psocoptera': Psocidae: Psocinae). Entomobrasilis, 10(2): 127-130.

García Aldrete, A.N. \& Mockford, E.L. 2009. A list of Psocoptera (Insecta: Psocodea) from Brazil. Revista Mexicana de Biodiversidad, 80: 665-673.

García Aldrete, A.N. \& Mockford, E.L. 2012. Psocoptera. In: Rafael, J.A.; Melo, G.A.R.; Carvalho C.J.B.; Casari, S.A. \& Constantino, R. Insetos do Brasil: Diversidade e Taxonomia, Brasil. São Paulo, Editora Holos. p. 423-437.

García Aldrete, A.N. \& Silva-Neto, A.M. 2013. Two new species of Lachesilla in the andra group, from the Chapada Diamantina, Bahia, Brazil (Psocodea: 'Psocoptera': Lachesillidae), Zootaxa, 3736(4): 392-396.

García Aldrete, A.N. \& Silva-Neto, A.M. 2014. Two new species of Lachesilla in species groups riegeli and forcepeta (Psocodea, 'Psocoptera', Lachesillidae), from the state of Bahia, Brazil. Revista Brasileira de Entomologia, 58(1): 7-10.

García Aldrete, A.N. \& Silva-Neto, A.M. 2019. A new Epipsocus (Psocodea: Psocomorpha: Epipsocidae) from the Brazilian Amazonia, with supernumerary forewing venation. Iheringia, Série Zoologia, 109: 1-3.

García Aldrete, A.N. \& Silva-Neto, A.M. 2020. New Neotropical species of Lachesilla Westwood, in species group forcepeta (Psocodea: 'Psocoptera': Lachesillidae). Zootaxa, 4728(1): 049-062.

García Aldrete, A.N.; Carrejo, N.S.; Mendivil, J.; Calderón, N.; Saenz, 0.; Román, C. \& González-Obando, R. 2018. Checklist of 'Psocoptera' (Psocodea) of Colombia and identification key to the families. Dugesiana, 25(2): 77-103.

García Aldrete, A.N.; González Obando, R.; Arenas-Clavijo, A. \& Lopes-Ferreira, R. 2016. Rediscovery of Antilopsocus nadleri Gurney, 1965 (Psocodea, Troctomorpha, Pachytroctidae) in South America. Check List, 12(5): 1979.

Johnson, K.P.; Yoshizawa, K. \& Smith, V.S. 2004. Multiple origins of parasitism in lice. Proceedings of the Royal Society of London, 271: 1771-1776.

Lienhard, C. 2016. Country checklists of the Psocoptera species of the World, extracted from Lienhard \& Smithers, 2002: Psocoptera (Insecta) - World Catalogue and Bibliography. Psocid News, 1: 1-123.

Lienhard, C. \& Lopes-Ferreira, R. 2013a. A new species of Neotrogla from Brazilian caves (Psocodea: 'Psocoptera': Prionoglarididae). Revue suisse de Zoologie, 120(1): 3-12.
Lienhard, C. \& Lopes-Ferreira, R. 2013b. Three new species of Psyllipsocus (Psocodea: 'Psocoptera': Psyllipsocidae) from Brazilian caves with description of a novel structure interpreted as a male accessory genital organ. Revue suisse de Zoologie, 120(3): 421-443.

Lienhard, C. \& Lopes-Ferreira, R. 2014. New species of Psyllipsocus from Brazilian caves (Psocodea: 'Psocoptera': Psyllipsocidae). Revue suisse de Zoologie, 121(2): 211-246.

Lienhard, C. \& Lopes-Ferreira, R. 2015. Review of Brazilian cave psocids of the families Psyllipsocidae and Prionoglarididae (Psocodea:'Psocoptera': Trogiomorpha) with a key to the South American species of these families. Revue suisse de Zoologie, 121(1): 121-142.

Lienhard, C. \& Smithers, C.N. 2002. Psocoptera (Insecta). World catalogue and Bibliography. Genève Muséum d'Histoire Naturelle. 745p. (Instrumenta Biodiversitatis V)

Lienhard, C.; Lopes-Ferreira, R.; Gnos, E.; Hollier, J.; Eggenberger, U. \& Piuz, A. 2012. Microcrystals coating the wing membranes of a living insect (Psocoptera: Psyllipsocidae) from a Brazilian cave. Scientific reports, 2: 1-6.

Lienhard, C.; Oliveira-Carmo, T. \& Lopes-Ferreira, R. 2010. A new genus of sensitibillini from Brazilian caves (Psocodea: 'Psocoptera': Prionoglarididae). Revue suisse de Zoologie, 117(4): 611-635.

Mockford, E.L. 2018. Biodiversity of Psocoptera. In: Foottit, R.G.\&Adler, P.H. Insect biodiversity: science and society. Hoboken, John Wiley \& Sons. p. 417-456.

Moura-Lima, D.; Silva-Neto, A.M.; García Aldrete, A.N. \& Bravo, F. 2019. Loneura Navás (Psocodea: Psocomorpha: Ptiloneuridae): new species from Brazil, and description of the female of Loneura maracaensis García Aldrete, with a checklist of all known species of the genus. Zootaxa, 4576(1): 179-186.

Rafael, J.A.; Melo, G.A.R.; Carvalho, C.J.B.; Casari, S.A. \& Constantino, R. 2012. Insetos do Brasil: diversidade e taxonomia. Brasil. São Paulo, Editora Holos. $796 \mathrm{p}$.

Román-P., C.; González Obando, R. \& García Aldrete, A.N. 2015. The genus Elaphopsocus Roesler (Psocodea: 'Psocoptera': Psocidae) with six new species from Brazil and Colombia. Zootaxa, 3957(2): 151-168.

Silva-Neto, A.M. \& García Aldrete, A.N. 2012. A new species of Spurostigma Eertmoed (Psocodea: 'Psocoptera': Spurostigmatidae) from Brazil. Zootaxa, 3501: 83-87.

Silva-Neto, A.M. \& García Aldrete, A.N. 2015. A new genus in the family Ptiloneuridae (Psocodea: 'Psocoptera': Psocomorpha: Epipsocetae) from Brazil. Zootaxa, 3914: 168-174.

Silva-Neto, A.M. \& García Aldrete, A.N. 2019. Triplocania Roesler, 1940: new record for Triplocania rosae Silva-Neto, García Aldrete \& Rafael, 2016 and proposal of a new infrageneric group (Psocodea, 'Psocoptera', Ptiloneuridae). Entomological Communications, 1: 1-3, e01005.

Silva-Neto, A.M.; Bravo, F. \& García Aldrete, A.N. 2013. Primeiro registro de Ectopsocus titschacki Jentsch (Psocodea: Psocoptera: Ectopsocidae) para 0 Estado da Bahia: uma prova da falta de estudos nessa Ordem de Insecta no Brasil. Entomobrasilis, 6(1): 86-88.

Silva-Neto, A.M.; Bravo, F. \& García Aldrete, A.N. 2014a. Psocídeos (Psocodea: 'Psocoptera') do Semiárido: uma diversidade subestimada. In: Bravo, F. \& Calor, A. Artrópodes do Semiárido. Biodiversidade e Conservação. Feira de Santana, Printmídia. p. 117-126.

Silva-Neto, A.M.; Rafael, J.A. \& García Aldrete, A.N. 2014b. New species of Triplocania Roesler with forewing M3 forked (Psocodea: 'Psocoptera': Ptiloneuridae), from Brazil. Zootaxa, 3838: 77-86.

Silva-Neto, A.M.; Rafael, J.A. \& García Aldrete, A.N. 2015. New species of Triplocania Roesler (Psocodea, 'Psocoptera', Ptiloneuridae), from Brazil and Ecuador. Zookeys, 505: 103-116.

Silva-Neto, A.M.; García Aldrete, A.N. \& Rafael, J.A. 2016a. Twelve new species of Triplocania Roesler (Psocodea: 'Psocoptera': Ptiloneuridae), from South America. Zootaxa, 4109: 251-283. 
Silva-Neto, A.M.; García Aldrete, A.N. \& Rafael, J.A. 2019b. New species of Euplocania Enderlein (Psocodea,'Psocoptera', Ptiloneuridae) from Brazil, with a checklist of all known species of the genus. Zootaxa, 4550(3): 374-390.

Smithers, C.N. 1991. Psocoptera (Psocids, Booklice). In: CSIRO (Ed.). The Insects of Australia. A textbook for students and research workers. 2.ed. New York, Cornell University Press. v. 1, p. 412-420.

Vinasco-Mondragón, A.F.; González-Obando, R. \& García Aldrete, A.N. 2018. The species group Amabilis of the genus Euplocania Enderlein (Psocodea: Psocomorpha: Ptiloneuridae). Zootaxa, 4444(1): 43-65.

Yoshizawa, K. \& Johnson, K.P. 2006. Morphology of male genitalia in lice and their relatives and phylogenetic implications. Systematic Entomology, 31: 350-361.

Yoshizawa, K. \& Johnson, K.P. 2010. How stable is the "Polyphyly of Lice" hypothesis (Insecta: Psocodea)?: A comparison of phylogenetic signal in multiple genes. Molecular Phylogenetics and Evolution, 55: 939-951.

Yoshizawa, K. \& Lienhard, C. 2010. In search of the sister group of the true lice: a systematic review of booklice and their relatives, with an updated checklist of Liposcelididae (Insecta: Psocodea). Arthropod Systematics and Phylogeny, 68: 181-195.

Yoshizawa, K.; Souza-Silva, M. \& Ferreira-Lopes, R. 2017. Disjunct occurrence of Trichadenotecnum s.str. in Southeastern Brazil (Psocodea: "Psocoptera": Psocidae), with description of a new species. Neotropical Entomology, 46: 169-174. 